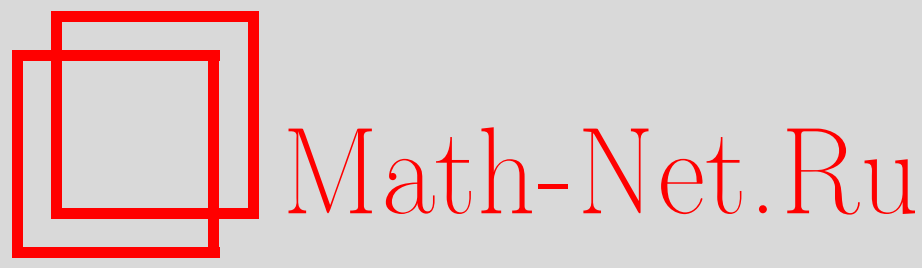

M. Wang, G. Wüstholz, Meromorphic Maps on Riemann Surfaces and Transcendence, Совр. пробл. матем., 2013, выпуск 17, 113-134

DOI: https://doi.org/10.4213/spm46

Использование Общероссийского математического портала Math-Net.Ru подразумевает, что вы прочитали и согласны с пользовательским соглашением http://www . mathnet.ru/rus/agreement

Параметры загрузки:

IP : 3.80 .181 .102

26 апреля 2023 г., 14:05:34

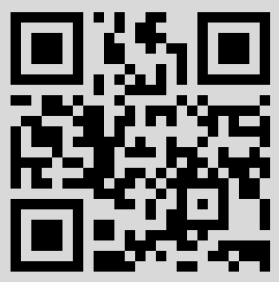




\title{
Meromorphic Maps on Riemann Surfaces and Transcendence
}

\author{
M. Wang, G. Wüstholz \\ Swiss Federal Institute of Technology, Switzerland
}

A. A. Karatsuba in großer Hochachtung gewidmet

\section{Introduction}

In this paper we prove a generalization of a theorem of Schneider which can be seen as the counterpart in the theory of transcendental numbers to work of Chern [1] in value distribution theory.

In the past transcendence theory has been largely related to the study of values of analytic functions with additional properties. The first remarkable instance was Hermite's work on $e$ followed by Lindemann's spectacular proof of the transcendence of $\pi$ as a corollary of his celebrated theorem on the transcendence of $e^{\alpha}$ for algebraic $\alpha \neq 0$. A further example is the solution of Hilbert's seventh problem by Gelfond [2] and independently by Schneider [3]. All these results are theorems about holomorphic functions of exponential type which satisfy linear differential equations. In 1936 Schneider [4] extended the results to elliptic functions which again satisfy a system of first order differential equations. In contrast to exponential functions they are only meromorphic and have order of growth 2. Then Schneider [5] realized in 1948 that the methods which were developed by himself and by Gelfond can be used to prove a very general and very conceptual transcendence criterion which included all the results described so far. Schneider pointed out in $[5 ; \S 3, \mathrm{~b})]$ that Gelfond's proof of the Hilbert problem is not covered by his theorem and he puts this as an open question. In his book Lang [6] streamlined the formulation of the theorem by simplifying the hypotheses and got a very elegant criterion, now known as the Schneider-Lang Criterion. This has the effect that the theorem becomes less general but has the advantage that its proof becomes slightly simpler and that the criterion is much easier to apply. In particular Lang assumes that the functions satisfy differential equations which is not needed in Schneider's theorem. However it still covers the main applications and even includes Gelfond's proof. It is clear that requiring differential equations is restrictive and schrinks the general applicability of the theorem.

SCHNEIDER-LANG CRITERION. Let $K \subset \mathbb{C}$ be a number field and let $f_{1}, \ldots, f_{N}$ be meromorphic functions of order $\leqslant \rho$. Assume that the field $K(f)=K\left(f_{1}, \ldots, f_{N}\right)$ has transcendence degree $\geqslant 2$ over $K$ and that the derivative $\nabla=d / d t$ maps the ring $K[f]=K\left[f_{1}, \ldots, f_{N}\right]$ into itself. If $\mathcal{S}$ is a set of points in $\mathbb{C}$ such that

$$
f_{i}(w) \in K
$$

for all $w \in \mathcal{S}$ then $|\mathcal{S}| \leqslant 20 \rho[K: \mathbb{Q}]$.

The elegance of the criterion was the starting point for further spectacular progress. In the same book [6] Lang got a version of the theorem for functions on $\mathbb{C}^{n}$. However he did not make real use of the much more complicated complex analysis in the case of several variables. Schneider had already pointed out among others in his paper this possibility. Later, in a wonderful paper [7], Bombieri, using very deep techniques in complex analysis of several variables, got the genuine several variables version of Schneider's theorem á la Schneider-Lang.

The first author was supported by scholarship of the Zurich Graduate School in Mathematics. 
Bombieri's Theorem. Let $K \subset \mathbb{C}$ be a number field and let $f=\left(f_{1}, \ldots, f_{n}\right)$ be meromorphic functions in $\mathbb{C}^{d}$ of finite order. Assume that

(1) $\operatorname{tr} \operatorname{deg} K(f) \geqslant d+1$;

(2) the partial derivatives $\partial / \partial z_{\alpha}, \alpha=1, \ldots, d$, map the ring $K[f]$ into itself.

Then the set $\mathcal{S}$ of points $\xi \in \mathbb{C}^{n}$ where $f(\xi)$ takes values in $K^{N}$ is contained in an algebraic hypersurface.

It is remarkable that the proofs of the two theorems above are closely related to value distribution theory in the case of functions on $\mathbb{C}$ and $\mathbb{C}^{n}$ respectively.

One main topic in Nevanlinna theory is to understand the relation between the growth of pole divisors and the growth of functions. In the case of rational functions the situation is very simple because the divisors are all finite and then the theory dates back to Gauss and his work on the fundamental theorem of algebra. In the case of meromorphic functions on $\mathbb{C}$ the value distribution theory is highlighted by two Main Theorems which can be found in the monograph [8] by Nevanlinna.

The beauty of Nevanlinna's theory seduced many mathematicians to try to understand the distribution property of functions in different and more general situations. First it was extended to functions on $\mathbb{C}^{n}$ by Stoll in [9] and by Chern [10]. Chern's insight into the role played by infinity in Nevanlinna theory lead to an extension of the theory to affine curves which was published in [1]. The general case of affine varieties has been accomplished by Ph. Griffiths and King in [11] and [12]. They introduced exhaustion functions to define growth and made use of differential geometric and complex algebrao-geometric methods.

The work of Schneider, Lang and Bombieri in transcendence theory is related to the cases $\mathbb{C}$ and $\mathbb{C}^{n}$ which were studied by Nevanlinna and Stoll whereas our work now deals with affine curves.

Let $Z$ be a smooth projective curve of genus $g$ over $\mathbb{C}$ and $\mathcal{P}$ a non-empty set of $l$ points in $Z$. Then $C:=Z-\mathcal{P}$ is an affine algebraic curve. We let $X$ be a non-singular algebraic variety defined over a number field $K$ with tangent sheaf $\mathcal{T}_{X}$. Further we assume that $\psi: C \rightarrow X_{\mathbb{C}}$ is an integral curve of a vector field $\Delta \in \Gamma\left(X, \mathcal{T}_{X} \otimes \mathcal{M}_{X}\right)$ which by definition acts on the sheaf of meromorphic sections $\mathcal{M}_{X}$ as derivations. Then for every $c \in C$ there is an analytic local section $\nabla$ at $c$ of the tangent sheaf $\mathcal{T}_{C}$ which does not vanish at $c$ and such that locally we have $\psi_{*}(\nabla)=\Delta_{\mathbb{C}}$. The section $\nabla$ acts on analytic functions on $C$ as derivation $f \mapsto \nabla(f):=(d f, \nabla)$ given by evaluating the differential $d f$ of a function $f$ in $\nabla$. The local sections $\nabla$ for varying $c \in C$ glue together and give a nowhere vanishing holomorphic global section $\nabla \in \Gamma\left(C, \mathcal{T}_{C}\right)$.

Our results depend on the order of the integral curve $\psi$. There are several essentially equivalent approaches to a concept of order in our situation. In Section 5 we shall discuss the different ways to define an order $\rho(\psi)$ of $\psi$ and we shall show that they lead to the same value which coincides with the order in the Schneider-Lang criterion when $C=\mathbb{A}^{1}(\mathbb{C})$ and $X=\mathbb{A}^{n}$.

THEOREM 1.1. If the dimension of the Zariski closure $\overline{\psi(C)}$ of $\psi(C)$ is at least 2 then

$$
\left|\psi^{-1}(X(K))\right| \leqslant g+1+2[K: \mathbb{Q}] l \max (\rho, 2 g) .
$$

In Theorem 1.1 we start from objects on $X$. If instead we start with objects on $C$ we get the following theorem which then takes more the form of the Schneider-Lang criterion.

THEOREM 1.2. Let $K \subset \mathbb{C}$ be a number field and let $f_{1}, \ldots, f_{N}$ be holomorphic functions on $C$ with order $\leqslant \rho$. We assume that the field $K\left(f_{1}, \ldots, f_{N}\right)$ has transcendence degree $\geqslant 2$ over $K$ and that $\nabla$ is an analytic section of $\mathcal{T}_{C}$ which acts as a derivation on $K\left[f_{1}, \ldots, f_{N}\right]$ by $f \mapsto(\nabla, d f)$. If $\mathcal{S}$ is a subset of $C$ such that $f_{i}(w) \in K$ for all $w \in \mathcal{S}$ and all $i$ then

$$
|\mathcal{S}| \leqslant g+1+2[K: \mathbb{Q}] \rho l .
$$

We give two proofs of the theorem in the last Section. One is based on a Jensen's Formula which will be discussed in Section 4 and a second uses the maximum principle. 
Before we state our next theorem we discuss how Theorem 1.1 and Theorem 1.2 are related. Let $f_{1}, \ldots, f_{N}$ be functions on $C$ as in Theorem 1.2 and let $F: C \rightarrow \mathbb{C}^{N}$ be the map $F(z)=\left(f_{1}(z), \ldots, f_{N}(z)\right)$. The derivation $\nabla$ of $K\left[f_{1}, \ldots, f_{N}\right]$ can be lifted to give a regular algebraic vector field $\Delta$ on $\mathbf{A}_{K}^{N}$ which can be obtained explicitly. For its construction we express $K\left[f_{1}, \ldots, f_{N}\right]$ as $K\left[T_{1}, \ldots, T_{N}\right] / I$ for an ideal $I$. If $\nabla\left(f_{i}\right)=g_{i}\left(f_{1}, f_{2}, \ldots, f_{N}\right)$ then we take $\Delta=\sum_{i} g_{i}\left(T_{1}, \ldots, T_{N}\right) \partial / \partial T_{i}$. Clearly the choice of $\Delta$ is not unique since taking $\Delta+h \partial / \partial T_{i}$ for any $h \in I$ is another possibility to select. It is clear however that their restrictions to $K\left[f_{1}, \ldots, f_{N}\right]$ coincide.

For $a \in C$ we denote by $\nabla_{a}$ the germ of $\nabla$ at $a$. We have $d F\left(\nabla_{a}\right)\left(T_{i}\right)=\nabla_{a}\left(F^{*}\left(T_{i}\right)\right)$ by the definition of $d F$, we have $\nabla_{a}\left(F^{*}\left(T_{i}\right)\right)=g_{i}(F(a))$ by hypothesis and we have $g_{i}(F(a))=\Delta_{F(a)}\left(T_{i}\right)$ by construction. Since the differentials $d T_{i}$ give a basis for $\Omega_{\mathbb{C}^{N}}^{1}$ at every point in $\mathbb{C}^{N}$ we deduce that $d F\left(\nabla_{a}\right)=\Delta_{F(a)}$ and we conclude that $F: C \rightarrow \mathbb{C}^{N}$ is an integral curve of $\Delta_{\mathbb{C}}$ provided that $\nabla$ does not have any zero on $C$. This shows that Theorem 1.2 is a special case of Theorem 1.1 when $X=\mathbb{A}_{K}^{N}$. However using a result of Griffiths the proof of Theorem 1.1 can be reduced to a situation as given in Theorem 1.2. This will be explained in Section 6 .

After we had finished a first version of this article we learned that there is a paper by Wakabayashi [13] on the same topic. His result is similar to our Theorem 1.2 with the difference that it applies for meromorphic functions and gives a different estimate for the cardinality of $\mathcal{S}$. It is difficult to compare the estimate given there with ours because his order is defined in a different way. The order we use is defined in an intrinsic way. The proofs rely on Jensen's equality which needs exhaustion functions. Wakabayashi builds on Green's functions. In our approach we use Riemann-Roch to construct a finite map $C \rightarrow \mathbb{C}$ and then pull back the distance function of $\mathbb{C}$ to obtain the so called Griffiths-King's exhaustion function on $C$.

We are now going to state our Main Theorem and consider as before a holomorphic mapping $f: C \rightarrow X_{\mathbb{C}}$ where $X$ is a projective variety defined over $K$. Let $\nabla$ be a vector field in $\Gamma\left(C, \mathcal{T}_{C}\right)$ without zero and assume that $\nabla$ acts as a derivation on the field $f^{*}(K(X))$. Then $\nabla$ can again be lifted to a rational vector field $\Delta$ on $X$, i.e. $\nabla \in \Gamma\left(C, \mathcal{T}_{C}\right) \cap \Gamma\left(C, f^{-1}\left(\mathcal{T}_{X} \otimes \mathcal{M}_{X}\right)\right)$. We call a point $x \in X$ a regular point of $\Delta$ if and only if $\Delta\left(\mathcal{O}_{X, x}\right) \subseteq \mathcal{O}_{X, x}$. Otherwise we call $x$ a pole of $\Delta$. The set of regular points of $\Delta$ is an open subvariety $\mathcal{U}$ of $X$. As usual we let $\overline{f(C)}$ be the Zariski closure of $f(C)$.

TheOrem 1.3 (MAIN). If $\operatorname{dim} \overline{f(C)} \geqslant 2$ then

$$
\left|f^{-1}(\mathcal{U}(K))\right| \leqslant g+1+2[K: \mathbb{Q}] l \max (\rho, 2 g) .
$$

A significant hypothesis in our theorems is the existence of differential equations. There are two aspects which should be mentioned in this context. The first concerns the growth. It seems to be possible that in some cases the condition on the growth of $f$ can be replaced by more accessible data related to the differential equations which determine the growth behavior of the solutions to some extend. We intend to come back to this question in the future.

The second concerns Schneider's original work where he taught us that differential equations are not necessary. They can also be removed from our work in the spirit of Schneider without making our work obsolete since results which can be expected without assuming differential equations are of different nature. We shall also come back to this point in the last section.

Our main Theorem 1.3 goes much beyond Theorem 1.1 and Theorem 1.2 and much beyond Wakabayashi's result since it deals with maps instead of functions and in addition - and this is a novelty - we allow singularities of the differential operator. To deal with singularities we use techniques from algebraic geometry and commutative algebra and use a blow up argument to reduce the singular case to a regular. In Section 7 we give several examples to illustrate how singular differential operators appear in nature. In particular we deduce an old theorem of Schneider on the transcendence of periods of elliptic functions. A similar example could also be given for abelian functions. 
Theorem 1.3 and Theorem 1.1 are more of geometric nature and can be seen as a finiteness Theorem on rational points on a certain class of analytic curves. Such an aspect is missing completely in the work of Wakabayashi.

Finally we should explain why we for the moment kept off from the higher dimensional question á la Bombieri. The main reason is that we intended first to investigate carefully all the possibilities which one has in a very new and unexplored area. For this we chose the most simple but still generic new situation. The next step would then be to extend the work to mappings from an affine variety into a projective variety along the lines given by Bombieri. The main work here consists of extending the $L^{2}$-analysis on $\mathbb{C}^{n}$ developed by Hörmander in [14] and used by Bombieri in his work to affine varieties. There are no fundamental obstructions to be expected, in particular since much work has been done in this direction so far by quite a number of authors, especially by H. Skoda and J.-P. Demailly.

Since Schneider's original theorem has stayed relatively unattended we decided to state and discuss the theorem in a version more in the style of today. We do this at the end of the paper in an appendix and we shall also discuss possible extensions.

\section{Standard Estimates}

Let $K$ be an algebraic number field of degree $d$ over the rationals $\mathbb{Q}$ and with discriminant $\operatorname{disc}(\mathrm{K})$. For a place $v$ of $K$ we denote by $|\cdot|_{v}$ the normalized absolute value such that $|p|_{v}=$ $p^{-\left[K_{v}: \mathbb{Q}_{p}\right]}$ when $v \mid p$ where $K_{v}$ is the completion of $K$ at $v$. For an archimedean place $v \mid \infty$ corresponding to the embedding $\tau$ of $K$ into $\mathbb{C}$ we define $|x|_{v}=|\tau(x)|^{\left[K_{v}: \mathbb{R}\right]}$ where $|\tau(x)|$ is the Euclidean absolute value and where $K_{v}$ is defined as in the non-archimedean case. Let $\mathbb{A}^{n}$ be the affine space of dimension $n$. As in [15] we introduce for $x=\left(x_{1} \ldots, x_{n}\right) \in \mathbb{A}^{n}(K)$ the absolute values $^{1}$

$$
|x|_{v}= \begin{cases}\max _{i}\left(\left|x_{i}\right|_{v}\right), & v \text { non-archimedean } \\ \left(\sum_{i} \tau\left(x_{i}\right)^{2}\right)^{1 / 2}, & v \text { real, } \\ \sum_{i} \tau\left(x_{i}\right) \bar{\tau}\left(x_{i}\right), & v \text { complex }\end{cases}
$$

and define the logarithmic (Weil) height $h_{L^{2}}(x)$ of $x \in \mathbb{A}^{n}(K)$ as

$$
h_{L^{2}}(x)=\sum_{v} \log |x|_{v}
$$

On writing $\log ^{+}(x)$ for $\max (0, \log (|x|))$ we introduce

$$
h_{L^{2}}^{+}(x)=\sum_{v} \log ^{+}|x|_{v}
$$

The two sums are taken over all places of $K$. The definition of the height depends on the field $K$ for which $x \in \mathbb{A}^{n}(K)$.

If we take instead of the $L^{2}$-norm the maximum norm $|x|_{v}=\max \left(\left|x_{i}\right|_{v}\right)$ we get a different height $h_{\max }(x)$. Both are related by

$$
h_{\max } \leqslant h_{L^{2}} \leqslant h_{\max }+\frac{d}{2} \log (n+1)
$$

\footnotetext{
${ }^{1}$ The absolute values are norms as soon as $v$ is either a non-archimedean or a real place. Norms are subadditive. At the remaining places only the square root of the absolute value is a norm. However on taking log ${ }^{+}$all absolute values lead to subadditive functions. The modified definition comes from the fact that there are $r$ real and $2 s$ complex embeddings but only $r$ real and $s$ complex places. This is compensated by introducing the weight $2=[\mathbb{C}: \mathbb{R}]$ at the non-real archimedean places and is built in in the definition of our absolute values so that the sum of the weights is $r+2 s$. This gives the very useful subadditivity $h_{L^{2}}(x+y) \leqslant h_{L^{2}}(x)+h_{L^{2}}(y)$ and the same for $h_{\max }$.
} 
when evaluated on $\mathbb{A}^{n}$. The inequalities also hold for $h_{L^{2}}$ and $h_{\max }$ repaced by $h_{L^{2}}^{+}$and $h_{\max }^{+}$. In the case $n=1$ we also introduce

$$
h(x)=\sum_{v} \log ^{+}|x|_{v}
$$

The norm satisfies the product formula

$$
\prod_{v}|x|_{v}=1
$$

for any $x \neq 0$ in $K$.

The Weil height can be extended to polynomials in $n$ variables $T_{1}, \ldots, T_{n}$ with coefficients in $K$. Let $P=\sum_{i} p_{i} T^{i}$ be such a polynomial with $i:\{1, \ldots, n\} \rightarrow \mathbb{N}$ a multi-index and $T^{i}=$ $T_{1}^{i(1)} \ldots T_{n}^{i(n)}$. It corresponds to a point $p=\left(\ldots, p_{i}, \ldots\right)$ in an affine space $\mathbb{A}^{N}(K)$ and we define $|P|_{v}$ as $|p|_{v}$ and the height of $P$ as

$$
h(P)=\sum_{v} \log |P|_{v}
$$

where $h$ is $h_{L^{2}}$ or $h_{\max }$ depending on the choice of norms. We shall also use

$$
h^{+}(P)=\sum_{v} \log ^{+}|P|_{v}
$$

The next Lemma is a modification of the classical Liouville estimate which gives a natural improvement in a special case.

Lemma 2.1 (Modified Liouville Estimate). Let $L=l_{1} T_{1}+\cdots+l_{N} T_{N}$ be a linear form with coefficients in $\mathbb{Z}, w$ is an archimedean place of $K$ and $\xi \in K^{N}$. If $L(\xi) \neq 0$ then

$$
\log |L(\xi)|_{w} \geqslant-\left(h_{L^{2}}^{+}(\xi)+h_{L^{2}}(L)\right)+\log |L|
$$

with $|L|$ the $L^{2}$-norm of $L$.

Proof. This is an easy application of the product formula and one has only to observe that by the Schwarz inequality we have $\log |L(\xi)|_{v} \leqslant \log |L|_{v}+\log |\xi|_{v}$ for an archimedean $v$ and $\log |L(\xi)|_{v} \leqslant$ $\log |\xi|_{v}$ in the non-archimedean case.

In the proofs we shall consider a system of linear equations

$$
L_{i}\left(T_{1}, \ldots, T_{N}\right)=\sum_{j=1}^{N} l_{i, j} T_{j}=0
$$

with coefficients in $K$ and with $1 \leqslant i \leqslant M$.

Lemma 2.2 (Siegel's Lemma). If $N>M$ then (4) has a non-trivial solution $x=\left(x_{1}, \ldots, x_{N}\right) \in$ $\mathcal{O}_{K}^{N}$ such that

$$
h_{\max }^{+}(x) \leqslant \frac{1}{2} \log |\operatorname{disc}(K)|+\frac{M}{N-M} \max _{i}\left(h_{L^{2}}\left(L_{i}\right)\right) .
$$

Proof. This is Corollary 11 of [15].

Let $\Delta: K\left[T_{1}, \ldots, T_{N}\right] \rightarrow K\left[T_{1}, \ldots, T_{N}\right]$ be a differential operator of order 1 . We may write

$$
\Delta=g_{1} \frac{\partial}{\partial T_{1}}+g_{2} \frac{\partial}{\partial T_{2}}+\cdots+g_{N} \frac{\partial}{\partial T_{N}}
$$


with $g_{1}, g_{2}, \ldots, g_{N}$ in $K\left[T_{1}, \ldots, T_{N}\right]$. It maps the vector space $K\left[T_{1}, \ldots, T_{N}\right]_{D}$ of polynomials of degree $\leqslant D$ into $K\left[T_{1}, \ldots, T_{N}\right]_{D+\omega}$ for $\omega=\max _{i}\left(\operatorname{deg} g_{i}\right)-1$ and we get for any set of integers $m_{j}$ with $0 \leqslant m_{j} \leqslant m$ in terms of the $L^{2}$-norm the bounds

$$
\log ^{+}\left|\Delta T_{1}^{m_{1}} \cdots T_{N}^{m_{N}}\right|_{v} \leqslant \begin{cases}\sum_{j} \log ^{+}\left|g_{j}\right|_{v}+\log ^{+}|m|_{v}+\log (N), & v \text { real, } \\ \sum_{j} \log ^{+}\left|g_{j}\right|_{v}+\log ^{+}|m|_{v}+\log (N), & v \text { complex, } \\ \max _{j}\left(\log ^{+}\left|g_{j}\right|_{v}\right), & v \text { archimedean. }\end{cases}
$$

Together with induction this leads to the following.

Lemma 2.3. Let $P$ be a polynomial of degree $\leqslant r$ in $K\left[T_{1}, \ldots, T_{N}\right]$ and $x \in K^{N}$. Then there exist positive real constants $C_{v}$ depending only on $\Delta, N$ and $x$ such that $C=\sum_{v} C_{v}$ is finite and

$$
\log ^{+}\left|\Delta^{k} P(x)\right|_{v} \leqslant \begin{cases}\log ^{+}|P|_{v}+k \log (r+k \omega)+C_{v}(r+k \omega), & v \text { real, } \\ \log ^{+}|P|_{v}+2 k \log (r+k \omega)+C_{v}(r+k \omega), & v \text { complex, } \\ \log ^{+}|P|_{v}+C_{v}(r+k \omega), & v \nmid \infty .\end{cases}
$$

REMARK. The constant $C$ can be easily seen to be of the form $C^{\prime} h^{+}(x)$ with $C^{\prime}$ independent on $x$.

\section{Rational Functions on Curves}

Let $Z$ be as in the introduction a smooth projective curve of genus $g, \mathcal{P}$ a non-empty finite set of $l$ points in $Z, \mathcal{S}$ a finite set of points in $C=Z \backslash \mathcal{P}$ and $\nabla \in \Gamma\left(C, \mathcal{T}_{C}\right)$. We shall construct in this section rational functions with prescribed zero and polar divisors using the classical theory of linear systems and the Riemann-Roch Formula. For a divisor $D$ we write $\mathcal{O}(D)$ for the invertible sheaf associated with $D$ which is $\mathcal{L}(D)$ in Hartshorne's notation. The first lemma is classical but for convenience we give the short proof.

Lemma 3.1. Let $Z$ be a compact Riemann surface of genus $g$ and let $D$ be a divisor on $Z$ with $\operatorname{deg} D \geqslant 2 g$. Then the complete linear system $|D|$ of $\mathcal{O}(D)$ has no base point.

Proof. For $p \in Z$ the exact sequence

$$
0 \rightarrow \mathcal{O}(D-(p)) \rightarrow \mathcal{O}(D) \stackrel{r_{p}}{\longrightarrow} L_{p} \rightarrow 0
$$

induces an exact sequence

$$
H^{0}(Z, \mathcal{O}(D)) \rightarrow H^{0}\left(Z, L_{p}\right) \rightarrow H^{1}(Z, \mathcal{O}(D-(p)))
$$

in cohomology. Since the canonical bundle $K_{Z}$ on $Z$ has degree $2 g-2$ and since $\operatorname{deg} \mathcal{O}(D-(p))=$ $\operatorname{deg} D-1 \geqslant 2 g-1$ we have $\operatorname{deg}\left(K_{Z} \otimes(\mathcal{O}(p)-D)\right)<0$ and Serre duality gives

$$
H^{1}\left(Z, \mathcal{O}(D-(p))=H^{0}\left(Z, K_{Z} \otimes \mathcal{O}((p)-D)\right)=0 .\right.
$$

We deduce that the connecting homomorphism $H^{0}(Z, \mathcal{O}(D)) \rightarrow H^{0}\left(Z, L_{p}\right)$ is surjective and this means that $p$ is not a base point and the conclusion of the lemma follows.

LEMmA 3.2. We assume that $|\mathcal{S}| \geqslant m_{1}|\mathcal{P}|+2 g$ for some $m_{1} \geqslant 1$. Then there exists a holomorphic map $f: Z \rightarrow \mathbb{P}^{1}$ such that $(f)_{0}=\sum_{w \in \mathcal{S}}(w)$ and $(f)_{\infty} \geqslant \sum_{p \in \mathcal{P}} m_{1}(p)$.

Proof. We apply the Riemann-Roch Formula to the divisor

$$
D=\sum_{w \in \mathcal{S}}(w)-\sum_{p \in \mathcal{P}} m_{1}(p)
$$


which has $\operatorname{deg} D=|\mathcal{S}|-m_{1} l \geqslant 2 g$ and get $l(D)=i(D)+\operatorname{deg} D+1-g \geqslant g+1$. The linear system $|D|$ is a projective space of dimension $g$. By Lemma 3.1 we know that $|D|$ has no base points. Therefore for $w \in \mathcal{S}$ the space $D_{w}=\left\{D^{\prime} \in|D|: w \in D^{\prime}\right\}$ is a hyperplane in $|D|$ and $|D|-\bigcup_{w \in \mathcal{S}} D_{w}$ is nonempty. Each $D^{\prime} \in|D| \backslash \bigcup_{w \in \mathcal{S}} D_{w}$ is effective and has the property that $D \sim D^{\prime}$. Therefore $\sum_{w \in \mathcal{S}}(w) \sim m_{1} \mathcal{P}+D^{\prime}$. By our selection of $D^{\prime}$ we have $w \notin D^{\prime}$ for $w \in \mathcal{S}$ and this implies that no $(w)$ with $w \in \mathcal{S}$ can be canceled by $D^{\prime}$. The difference is linearly equivalent to zero and this means that there is a holomorphic map $f: Z \rightarrow \mathbb{P}^{1}$ such that $(f)_{0}=\sum_{w \in \mathcal{S}}(w)$ and $(f)_{\infty}=\sum_{p \in \mathcal{P}} m_{1}(p)+D^{\prime} \geqslant \sum_{p \in \mathcal{P}} m_{1}(p)$ as stated.

For each $p \in \mathcal{P}$ we choose a local coordinate $z_{p}$ in a neighborhood of $p$. We assume that $|\mathcal{S}| \geqslant|\mathcal{P}|+2 g$ and then there exist integers $m_{1} \geqslant 1$ and $2 g \leqslant t \leqslant l+2 g-1$ such that $|\mathcal{S}|=$ $m_{1}|\mathcal{P}|+t$.

Lemma 3.3. There exist constants $C_{1}, C_{2}>0$ such that for all integers $N \geqslant 1$ there is a holomorphic mapping $\phi_{N}: Z \rightarrow \mathbb{P}^{1}$ such that

(i) $\left(\phi_{N}\right)_{0}=\sum_{w \in \mathcal{S}} N(w)$;

(ii) $\left|\phi_{N}\left(z_{p}\right)\right| \geqslant\left|C_{1} / z_{p}\right|^{m_{1} N}$ for all $p \in \mathcal{P}$ and all $z_{p}$ sufficiently small;

(iii) $\left|\nabla^{N} \phi_{N}(w)\right| \leqslant N ! C_{2}^{N}$ for all $w \in \mathcal{S}$.

Proof. By Lemma 3.2 there exists a function $g$ such that,

$$
(g)_{0}=\sum_{w \in \mathcal{S}}(w), \quad(g)_{\infty} \geqslant \sum_{p \in \mathcal{P}} m_{1}(p) .
$$

Now we put $\phi_{N}=g^{N}$ and (i) follows.

Since $g$ has a pole of order at least $m_{1}$ at each $p$ in $\mathcal{P}$ there exists a constant $C_{1}>0$ such that $\left|g\left(z_{p}\right)\right| \geqslant\left|C_{1} / z_{p}\right|^{m_{1}}$ for $z_{p}$ sufficiently small and $p \in \mathcal{P}$. Therefore the function $\phi_{N}$ can be estimated from below by $\left|\phi_{N}\left(z_{p}\right)\right| \geqslant\left|C_{1} / z_{p}\right|^{m_{1} N}$ for $z_{p}$ sufficiently small and $p \in \mathcal{P}$ which gives (ii).

Since $\nabla$ is non-zero at $w$ by hypothesis we find a local parameter $t$ at $w \in \mathcal{S}$ satisfying $t(w)=0$ such that in a neighborhood of $w$ the derivation $\nabla$ takes the form $\partial / \partial t$. Then $g(t)$ can be written as $t \epsilon(t)$ near $w$ for some unit $\epsilon(t)$ and $\phi_{N}(t)$ as $t^{N} \epsilon(t)^{N}$. Therefore $\nabla^{N} \phi_{N}(w)=N ! \epsilon(0)^{N}$ and if we define $C_{2}$ as the maximum of $|\epsilon(0)|$ taken over all $w \in \mathcal{S}$ we get (iii).

REMARK. The existence of $\phi_{N}$ in Lemma 3.3 is essential for the first proof of Theorem 1.2. In classical transcendence proofs there already exist functions which are analogous to our $\phi_{N}$. We mention the polynomial $\prod_{w \in \mathcal{S}}(t-w)^{N}$ in [6] or the Blaschke products $\prod_{w \in \mathcal{S}}\left(r(w-t) /\left(r^{2}-\bar{w} t\right)\right)^{N}$ in [16] which play the role of our $\phi_{N}$ there. All such $\phi_{N}$ have the property that they take 0 up to order $N$ at fixed finitely many points and take large values near the boundary.

In the second proof of Theorem 1.2 we need a special exhaustion function for the affine curve $C=Z \backslash \mathcal{P}$. This is provided in the next lemma.

Lemma 3.4. For all $a \in C$ and for all integers $q \geqslant 0$ there exists a rational map $\pi: Z \rightarrow \mathbb{P}^{1}$ such that

(i) $(\pi)_{0}=t(a), q l \leqslant t \leqslant q l+g$;

(ii) $(\pi)_{\infty} \geqslant \sum_{p \in \mathcal{P}} q(p)$.

The projection $\pi$ only depends on $a$ and $q$.

Proof. The divisor $D=(q l+g)(a)-\sum_{p \in \mathcal{P}} q(p)$ has degree $g$ and the Riemann-Roch Formula gives $l(D)=i(D)+\operatorname{deg} D+1-g \geqslant 1$ which implies the desired conclusion.

\section{Jensen's Formula}

In this section we shall discuss Jensen's Formula which was a starting point for Nevanlinna theory. Although this formula can be stated in very simple and elementary terms it is conceptually 
better and advantageously to express the formula in terms of the standard functions in Nevanlinna theory. We begin with recalling the definition of the Nevanlinna characteristic function and of the order of a meromorphic function $f$ on $\mathbb{C}$ and we shall state the First Main Theorem (FMT) in classical Nevanlinna theory which shows how the various functions are related.

For real $\alpha$ we recall $\log ^{+} \alpha$ to be the maximum of the numbers $\log \alpha$ and 0 , and we let $n(r, \infty)$ be the number of poles of $f$ in the closed disk $|z| \leqslant r$. Then the standard functions in Nevanlinna theory are

$$
\begin{aligned}
M(r, f) & =\max _{|z|=r}|f(z)| \\
N(r, f)=N(r, \infty) & =\int_{0}^{r} \frac{n(t, \infty)-n(0, \infty)}{t} d t+n(0, \infty) \log r \\
m(r, f)=m(r, \infty) & =\frac{1}{2 \pi} \int_{0}^{2 \pi} \log ^{+}\left|f\left(r e^{i \varphi}\right)\right| d \varphi \\
N(r, a) & =N\left(r, \frac{1}{f-a}\right) \\
m(r, a) & =m\left(r, \frac{1}{f-a}\right) \\
T(r, f) & =m(r, \infty)+N(r, \infty) .
\end{aligned}
$$

We call $M(r, f)$ the maximum modulus function and $T(r, f)$ the Nevanlinna characteristic function of $f$ and we write simply $M(r), m(r), N(r), T(r)$ when no confusion can arise.

First Main TheOREm [8; p. 166]. For any meromorphic function $f$ we have

$$
N(r, a)+m(r, a)=T(r, f)+O(1) .
$$

It is not difficult to see that the First Main Theorem is equivalent to Jensen's Formula which we shall present only for holomorphic functions since we need it only in this case. Such an entire function can be expressed as $f(z)=z^{\lambda} \epsilon(z)$ for some unit $\epsilon(z)$.

Jensen's Formula [8; p. 164]. We have

$$
\log |\epsilon(0)|+N(r, 0)=\frac{1}{2 \pi} \int_{0}^{2 \pi} \log \left|f\left(r e^{i \theta}\right)\right| d \theta .
$$

Jensen's Formula is closely related to the Schwarz Lemma as it is used in transcendence theory. There usually a holomorphic function is constructed with growth and vanishing conditions. The growth conditions are used to give an upper bound for the integral in the formula. The vanishing conditions lead to a lower bound for $N(r, 0)$. Arithmetical data enter through the term $\log |\epsilon(0)|$ on the left and one derives a lower bound of $\log |\epsilon(0)|$ using Liouville estimates. In this way a proof of Schneider's theorem can be obtained, although there is not too much difference with the standard proof. We shall give one proof of Theorem 1.2 along these lines. This needs a Jensen's Formula in the more general situation of an affine algebraic curve which we shall derive now.

Let $f$ be a meromorphic function on a smooth affine algebraic curve $C$ as in Section 3 . We recall briefly from [12] some aspects of Nevanlinna theory of affine varieties in the case of curves, especially the use of a special exhaustion function. Since $Z$ is a smooth curve we can choose by Riemann-Roch a projection $\pi: Z \rightarrow \mathbb{P}^{1}$ such that $\pi^{-1}(\infty)=\sum_{p \in \mathcal{P}} m_{p}(p)$ with $m_{p} \geqslant 1$. The projection $\pi$ gives a special exhaustion function $\tau(z)=\log |\pi(z)|$ on $C$ in the sense of Griffiths and King and for real $r \geqslant 0$ we put $C[r]=\left\{z \in C: e^{\tau(z)}=|\pi(z)| \leqslant r\right\}$. We consider now the curve $C$ as a Riemann surface and for real $r \geqslant 0$ we define Div $C[r]$ to be the free abelian group generated by $C[r]$. Its elements can be expressed as finite suns $\sum_{z \in C[r]} n_{z}(z)$ with integer coefficients $n_{z}$ and 
with a symbol $(z)$ for each $z \in C[r]$. For $s \leqslant r$ there is a natural injective group homomorphism $\iota_{s, r}: \operatorname{Div} C[s] \rightarrow \operatorname{Div} C[r]$.

The family $\left\{\operatorname{Div} C[r], \iota_{s, r}\right\}$ is a direct system and $\operatorname{Div} C=\underline{\lim } \operatorname{Div} C[r]$ is defined to be the group of analytic divisors on $C$. Its elements can be written as

$$
D=\sum_{z \in C} D(z)(z)
$$

with $D(z) \in \mathbb{Z}$ and zero up to a discrete and countable set of points, the support $\operatorname{supp}(D)$ of $D$. Let $p_{r}: \operatorname{Div} C \rightarrow \operatorname{Div} C[r]$ be the natural projection. Then $D[r]=p_{r}(D)$ has finite support in $C[r]$ and therefore $n^{\pi}(D, r)=\int_{D[r]} 1$ is an integer. To characterize the growth of $D$ we define

$$
N^{\pi}(D, r)=\int_{0}^{r} \frac{n^{\pi}(D, t)-n^{\pi}(D, 0)}{t} d t+n^{\pi}(D, 0) \log r .
$$

Let $f: C \rightarrow \mathbb{P}^{1}$ be a meromorphic function and $\operatorname{ord}_{z} f$ its order at $z \in C$. Then

$$
(f)=\sum_{z \in C}\left(\operatorname{ord}_{z} f\right)(z)
$$

is the divisor of $f$. To characterize the growth of the function $f$ we define the Ahlfors-Shimizu characteristic function by

$$
T_{A S}^{\pi}(r, f)=\int_{0}^{r} \int_{C[t]} f^{*}\left(\frac{i}{2 \pi} \frac{d z \wedge d \bar{z}}{\left(1+|z|^{2}\right)^{2}}\right) \frac{d t}{t}
$$

as given by (5.1) in [12] in the case $m=q=1$. From the definition we easily see that $T_{A S}^{\pi}(r, f) \geqslant$ $C \log r$ for some positive $C$ and sufficiently large $r$ if $f$ is non-constant. When we compare the growth of a divisor and the growth of a function, the FMT in Nevanlinna theory (see [1; p. 332] and [12; pp. 184, 189-190] for more details) shows us that the error term can be estimated by the proximity function

$$
m_{A S}^{\pi}(r, f)=\int_{\partial C[r]} \log \left(1+|f(z)|^{2}\right) d^{c} \tau
$$

with $d^{c} f$ defined as

$$
\frac{\sqrt{-1}}{4 \pi}\left(\frac{\partial f}{\partial z} d z-\frac{\partial f}{\partial \bar{z}} d \bar{z}\right)
$$

First MAIn TheOREM*. Let $D_{\infty}$ be the polar divisor of $f$. Then

$$
T_{A S}^{\pi}(r, f)=N^{\pi}\left(D_{\infty}, r\right)+m_{A S}^{\pi}(r, f)+O(1) .
$$

It can be shown that $m_{A S}^{\pi}(\infty, r)=\int_{\partial C[r]} \log ^{+}|f(z)|^{2} d^{c} \tau+O(1)$ and therefore, if we define the Nevanlinna's characteristic function $T^{\pi}(r, f)$ as $N^{\pi}\left(D_{\infty}, r\right)+m^{\pi}(r, f)$, where

$$
m^{\pi}(r, f)=\int_{\partial C[r]} \log ^{+}|f(z)|^{2} d^{c} \tau,
$$

we see that the First Main Theorem* implies that the Nevanlinna characteristic function and the Ahlfors-Shimizu characteristic function coincide up to a bounded term.

As we have already mentioned the FMT is equivalent to a formula of Jensen's type. We shall state now a version of a general Jensen Formula as given in [12; Proposition 3.2], that is adapted to our situation. 
JENSEN'S FORMUlA*. Let $f$ be a meromorphic function on $C$ with divisor $D$. Then for all real numbers $r$ and $r_{0}$ with $r \geqslant r_{0}$ we have

$$
N^{\pi}(D, r)-N^{\pi}\left(D, r_{0}\right)+\int_{\partial C\left[r_{0}\right]} \log |f|^{2} d^{c} \tau=\int_{\partial C[r]} \log |f|^{2} d^{c} \tau .
$$

An explicit form of FMT for functions on affine curves goes back to Chern in [1; p. 332]. Later Griffiths and King [12] were able to extend it to affine varieties using special exhaustion functions. Since Chern's result does not depend on any special exhaustion function his result is more general than the result of Griffiths and King in the one variable case. However we still prefer their setting because special exhaustion functions make the formula more applicable. When $n(D, 0)=0$ we can take $r_{0}=0$ and then the above formula becomes

$$
N^{\pi}(D, r)+\sum_{\pi(z)=0} \log |f(z)|=\int_{\partial C[r]} \log |f|^{2} d^{c} \tau .
$$

In the second proof of Theorem 1.2 we construct a holomorphic function $f$ which satisfies again growth and vanishing conditions. Then from the growth conditions we derive an upper bound for the integral. The vanishing conditions lead to a lower bound for $N^{\pi}(D, r)$ and the sum can be bounded from below by a Liouville estimate. A comparison of the bounds then leads to the stated result. In the next section we discuss the notion of the order of a function which is used to characterize growth conditions.

\section{Functions of finite order on curves}

Since there are at least two possible definitions of the order of functions on curves (see [17] or [12]) we have to discuss the notion carefully. For a clear and detailed exposition on the different indicators of orders for meromorphic functions $f: \mathbb{C} \rightarrow \mathbb{P}^{1}$ we refer to [18].

We discuss first the case when $f$ is an entire function. Here the order of $f$ is defined using the maximum modulus function or the Nevanlinna characteristic function. It is given by

$$
\rho(f)=\varlimsup_{r \rightarrow \infty} \frac{\log \log M(r, f)}{\log r}
$$

and

$$
\rho(f)=\varlimsup_{r \rightarrow \infty} \frac{\log T(r, f)}{\log r}
$$

respectively. The two definitions are equivalent and for a proof we refer to [8; p. 216].

When $f$ is a meromorphic function, $M(r, f)$ does not make sense. There are two variants to overcome the difficulty. The first makes use of the well-known fact that a meromorphic function is of order $\leqslant \rho$ if and only if it can be expressed as $f=h / g$ where $h$ and $g$ are both entire functions and of order $\leqslant \rho$ (see [8; p. 223]). Then $\rho(f)$ is the infimum of $\max (\rho(h), \rho(g))$ with $h$ and $g$ taken over all representations of $f=h / g$ as a quotient of two holomorphic functions. The second variant uses the Nevanlinna characteristic function $T(r, f)$ which is also well-defined for meromorphic functions.

In the case of a meromorphic function on a curve $C=Z \backslash \mathcal{P}$ we again begin with a holomorphic function $f$ and define the local maximum modulus function and the local order of $f$ at $p \in \mathcal{P}$ by

$$
M_{p}(r)=\max _{\left|z_{p}\right|=1 / r}\left|f\left(z_{p}\right)\right|, \quad \rho_{p}(f)=\varlimsup_{r \rightarrow \infty} \frac{\log \log M_{p}(r)}{\log r} .
$$

Then the order of $f$ is given by $\rho(f)=\max _{p \in \mathcal{P}} \rho_{p}(f)$ and it is easily seen that $\rho_{p}(f)$ and therefore $\rho(f)$ are independent of the choice of local coordinates. Moreover $f$ is of order $\rho$ if and only if 
$\rho$ is maximal with the property that for any $\epsilon>0$ we always have $\log \left|f\left(z_{p}\right)\right| \leqslant 1 /\left|z_{p}\right|^{\rho+\epsilon}$ for all $z_{p}$ sufficiently small. When $f$ is a meromorphic function we define $\rho_{p}(f)$ as before to be the infimum of $\max (\rho(h), \rho(g))$ taken over all representations of $f=h / g$ in a neighborhood of $p$ and $\rho(f)=\max \rho_{p}(f)$. This definition was suggested by Griffiths in [17].

Another approach to the growth of functions is to use Nevanlinna's or Ahlfors-Shimizu's characteristic function $T^{\pi}(r, f)$ and $T_{A S}^{\pi}(r, f)$ respectively and using a special exhaustion $\pi$ which was suggested by Griffiths and King in [12]. As already noted both functions coincide up to $O(1)$. Therefore we can use either of them to define the order of growth of a function $f$. We use Nevanlinna's characteristic function and put

$$
\rho_{T}^{\pi}(f)=\varlimsup_{r \rightarrow \infty} \frac{\log T^{\pi}(r, f)}{\log r} .
$$

When $f$ is holomorphic we can also use the maximum modulus function

$$
M^{\pi}(r, f)=\max _{z \in \partial C[r]}|f(z)|
$$

and get

$$
\rho^{\pi}(f)=\varlimsup_{r \rightarrow \infty} \frac{\log \log M^{\pi}(r, f)}{\log r} .
$$

Although it is not the main purpose of this paper we shall prove that $\rho_{T}^{\pi}(f)$ and $\rho^{\pi}(f)$ are equal. This gives a generalization of the classical identity for holomorphic functions on $\mathbb{C}$ described at the beginning of this section. It also provides an example how the exhaustion function of Griffith and King can be applied.

We begin with a simple lemma. Let $r: \mathbb{D} \rightarrow \mathbb{D}$ be the ramified covering of the open unit disk $\mathbb{D}$ of degree $n$ given by $z \mapsto z^{n}$ and assume that $u: \mathbb{D} \rightarrow \mathbb{R} \cup\{-\infty\}$ is a subharmonic function on $\mathbb{D}$.

LEMma 5.1. The function $v: \mathbb{D} \rightarrow \mathbb{R} \cup\{-\infty\}$ given by

$$
v(z)=\max _{r(w)=z} u(w)
$$

is subharmonic.

Proof. The function $v$ is upper semi-continuous and takes values in $[-\infty,+\infty)$. According to [19; Chap. 3, Corollary 7 to Proposition 4] the property to be subharmonic is local. To show that $v(z)$ is subharmonic we let $h(z)$ be a harmonic function on a sufficiently small compact disk $\mathbb{B} \subset \mathbb{D}$ with $h(z) \geqslant v(z)$ on the boundary $\partial B$. We shall prove that this holds for all $z$ in $\mathbb{B}$. This will imply that $v$ is subharmonic (see [19; Definition 4]).

The upper semi-continuous function $\varphi(w)=\max _{\zeta^{n}=1} u(\zeta w)$ is the maximum of a finite collection of subharmonic functions and therefore subharmonic. The pullback of $h(z)$ by the holomorphic function $r(w)$ is harmonic as one sees by applying the Laplace operator $4 \partial \bar{\partial}$ to the pullback. ${ }^{2}$ Furthermore it satisfies

$$
\left(r^{*} h\right)(w)=h(z) \geqslant v(z)=\max _{r(w)=z} u(w)=\max _{\zeta^{n}=1} u(\zeta w)=\varphi(w)
$$

for $w$ on the boundary $r^{-1}(\partial \mathbb{B})$ and $z=r(w)$. Since $\varphi(w)$ is subharmonic we deduce that this holds for all $w$ with $r(w) \in \mathbb{B}$. The image $z=r(w)$ of such a $w$ is in $\mathbb{B}$ and

$$
h(z)=\left(r^{*} h\right)(w) \geqslant \varphi(w)=\max _{\zeta^{n}=1} u(\zeta w)=\max _{r(w)=z} u(w)=v(z) .
$$

\footnotetext{
${ }^{2}$ Here the calculation: we write $u=u(w, \bar{w}), w=f(z), \bar{w}=\bar{f}(\bar{z})$ and differentiate. Applying first $\bar{\partial}$, then $\partial$ and observing that $\partial_{\bar{z}} f=0$ and $\partial_{z} \partial_{\bar{z}} f=0$ we get $\bar{\partial}\left(f^{*} u\right)=f^{*}\left(\partial_{w} u\right) \cdot \partial_{\bar{z}} \bar{f}$ and then

$$
\partial \bar{\partial}\left(f^{*} u\right)=f^{*}\left(\partial_{w} \partial_{\bar{w}} u\right) \cdot \partial_{z} f \partial_{\bar{z}} \bar{f}+f^{*}\left(\partial_{\bar{w}} u\right) \cdot \partial_{z} \partial_{\bar{z}} f=f^{*}\left(\partial_{w} \partial_{\bar{w}} u\right) \cdot \partial_{z} f \partial_{\bar{z}} \bar{f}=0 .
$$

Since $\partial_{z} f \partial_{\bar{z}} \bar{f} \geqslant 0$ we deduce - this only as a side remark - that the pullback of a subharmonic function by a holomorphic function is subharmonic. For this one uses that $\partial \bar{\partial} u \geqslant 0$ for subharmonic $u$.
} 
The lemma is applied to yield a global version for finite (i.e., proper and non-constant) mapping between Riemann surfaces.

LEMMA 5.2. For all finite and holomorphic mappings $f: X \rightarrow Y$ between Riemann surfaces and all subharmonic functions $u$ on $X$ the function $v(z)=\max _{f(w)=z} u(w)$ is subharmonic on $Y$.

Proof. Again we use that subharmonicity is a local property. The map $f$ is a finite covering and if the disk $i: \mathbb{D} \hookrightarrow Y$ is small enough the inverse image $f^{-1}(\mathbb{D})=\mathbb{D} \times_{Y} X$ of $\mathbb{D}$ under $f$ has only finitely many connected components $X_{\iota}$ and all ramification points are in the fiber over the center of the disk. The restriction $f_{\iota}$ of $f$ to any of the components $X_{\iota}$ then takes the form as described in Lemma 5.1 which we apply now to all the components. It follows that the function $v_{\iota}$ associated with $f_{\iota}$ is subharmonic. We write $v(z)$ as $\max _{\iota} v_{\iota}(z)$. The same arguments as in the proof of the previous lemma then show that $v(z)$ is subharmonic as stated.

REMARK. Our lemma can be regarded as subharmonic descent under holomorphic coverings and $v(z)$ can be seen as $\left(f_{*} u\right)(z)$. The Lemma then says that $f_{*} u$ is subhamonic for $u$ subharmonic.

We apply the Lemma in the case when $X$ is an affine curve and when $Y$ is the complex plane $\mathbb{C}$.

Proposition 5.1. We have

$$
\rho^{\pi}(f)=\rho_{T}^{\pi}(f)
$$

Proof. Let $d$ be the degree of $\pi$. Since $f$ is holomorphic, $N^{\pi}\left(D_{\infty}, r\right)$ is zero and therefore, by the definition of the Nevanlinna characteristic function, we have

$$
T^{\pi}(r, f)=m^{\pi}(r, f)=\int_{\partial C[r]} \log ^{+}|f(z)|^{2} d^{c} \tau \leqslant d \log ^{+} M^{\pi}(r) .
$$

We know that $\log ^{+}|f(w)|$ is subharmonic on $C$ for $f$ holomorphic and Lemma 5.2 implies that $h(z)=\max _{\pi(w)=z}\left(\log ^{+}|f(w)|\right)$ is a subharmonic function on $\mathbb{C}$. For $z=r e^{i \varphi}$ and $r<\varrho$ the Harnack inequality gives

$$
h(z) \leqslant \frac{\varrho+r}{\varrho-r} \int_{0}^{2 \pi} h\left(\varrho e^{i \theta}\right) d \theta \leqslant \frac{\varrho+r}{\varrho-r} \int_{\partial C[\varrho]} \log ^{+}\left(|f(z)|^{2}\right) d^{c} \tau=\frac{\varrho+r}{\varrho-r} T^{\pi}(\varrho, f)
$$

and this implies that

$$
\log ^{+} M^{\pi}(r, f) \leqslant \frac{\varrho+r}{\varrho-r} T^{\pi}(\varrho, f) .
$$

On putting the inequalities together we deduce that

$$
\frac{1}{d} T^{\pi}(r, f) \leqslant \log ^{+} M^{\pi}(r, f) \leqslant \frac{\varrho+r}{\varrho-r} T^{\pi}(\varrho, f) .
$$

Since $f$ is nontrivial, there exists $C>0$ such that $T^{\pi}(r, f) \geqslant C \log r$ (see the remark following (6) and notice that $T^{\pi}(r, f)$ and $T_{A S}^{\pi}(r, f)$ differ only by a bounded term). Therefore the left hand side of the inequalities shows that $\log ^{+} M^{\pi}(r, f)=\log M^{\pi}(r, f)$ for sufficiently large $r$. We put $\varrho=$ $r+\epsilon$ for $\epsilon>0$ and then an easy calculation gives

$$
\rho^{\pi}(f)=\varlimsup_{r \rightarrow \infty} \frac{\log \log ^{+} M^{\pi}(r)}{\log r}=\varlimsup_{r \rightarrow \infty} \frac{\log T^{\pi}(r, f)}{\log r}=\rho_{T}^{\pi}(f) .
$$

The order $\rho_{T}^{\pi}$ depends on the choice of the covering map $\pi$ in Section 4 whereas the order $\rho=$ $\max _{p} \rho_{p}$ with $p \in \mathcal{P}$ is independent of any choice. However they can be compared.

LEMмA 5.3. We have $\rho_{T}^{\pi}=\max _{p}\left(\rho_{p} / m_{p}\right)$. 
Proof. Since we shall not use Lemma 5.3 later we only verify it for holomorphic $f$. The function $(1 / \pi(z))^{1 / m_{p}}$ gives a local coordinate on a neighborhood $U_{p}$ of $p$ and then for sufficiently large $r$ we have

$$
M_{p}(r)=\max \left(|f(z)|: z \in U_{p},|\pi(z)|=r^{m_{p}}\right) .
$$

Since $M^{\pi}(r)=\max _{|\pi(z)|=r}(|f(z)|)$ we conclude that $M^{\pi}(r)=\max _{p} M_{p}\left(r^{1 / m_{p}}\right)$. This implies that

$$
\rho_{T}^{\pi}(f)=\rho^{\pi}(f)=\varlimsup_{r \rightarrow \infty} \frac{\log \log M^{\pi}(r)}{\log r}=\max _{p}\left(\varlimsup_{r \rightarrow \infty} \frac{\log \log M_{p}\left(r^{1 / m_{p}}\right)}{\log r}\right)
$$

and the latter is $\max _{p}\left(\rho_{p} / m_{p}\right)$.

We point out that $\rho(f)$ depends only on some smooth completion of $C$ and therefore is an intrinsic notion in the case of affine curves (since a smooth completion of a smooth affine curve is unique). However the order function $\rho^{\pi}$ depends on a special exhaustion function induced by a projection $\pi: C \rightarrow \mathbb{C}$ and is therefore an extrinsic notion. By Lemma 5.3 the order function $\rho(f)$ is finite if and only if $\rho^{\pi}(f)$ is finite and Griffiths uses both in [17] and [12]. However for our purpose an estimate in terms of $\rho(f)$ is essential.

Let $C$ be an affine algebraic curve and let $V$ be a projective algebraic variety. Let as usual $\mathfrak{R}(V)$ be the field of rational functions on $V$. A holomorphic map $f: C \rightarrow V$ has order $\leqslant \rho$ if and only if $f^{*} \mathfrak{R}(V)$ consists of meromorphic functions of order $\leqslant \rho$. It is easy to see that the latter is equivalent to $\rho\left(f^{*}\left(z_{i} / z_{j}\right)\right) \leqslant \rho$.

In the next proposition we shall compare the growth of a positive divisor $D=\sum_{z \in C} D(z)(z)$ with the growth of a function. We put

$$
n(r, D)=\sum_{z} D(z)
$$

where the sum is take over all $z \in C$ not in the union of the sets $\left|z_{p}\right| \leqslant r^{-1}$ with $p \in \mathcal{P}$ and

$$
\rho(D)=\varlimsup_{r \rightarrow \infty} \frac{\int_{r_{0}}^{r} n(t, D) d t / t}{\log r} .
$$

The following proposition is [17; Proposition 5.23].

Proposition 5.2. Let $D$ be a positive divisor on $C$. Then there exists a function $f \in \mathcal{O}(D)$ such that $(f)=D$ and

$$
\rho(f) \leqslant \max (\rho(D), 2 g) .
$$

It is essential for the proof of the following.

Lemma 5.4. Let $f_{1}, \ldots, f_{n}$ be meromorphic functions of order $\leqslant \rho$ on $C$ and let $\mathcal{S} \subset C$ be a finite set. If $f_{i}(w) \neq \infty$ for $1 \leqslant i \leqslant n$ and $w \in \mathcal{S}$ then there exists a holomorphic function $h$ on $C$ such that

(i) $\rho(h) \leqslant \max (\rho, 2 g)$;

(ii) $h f_{i}$ are all holomorphic and $\rho\left(h f_{i}\right) \leqslant \max (\rho, 2 g)$ for $1 \leqslant i \leqslant n$;

(iii) $h\left(w_{i}\right) \neq 0$ for $w \in S$.

Proof. By $(7)$ we have $\rho\left(\left(f_{i}\right)_{\infty}\right) \leqslant \rho$ and this gives

$$
\rho\left(\left(f_{1}\right)_{\infty}+\cdots+\left(f_{n}\right)_{\infty}\right) \leqslant \max _{i} \rho\left(f_{i}\right)_{\infty} \leqslant \rho .
$$

By Proposition 5.2 there is a holomorphic function $h$ of order at most $\max (\rho, 2 g)$ on $C$ such that $(h)=\left(f_{1}\right)_{\infty}+\cdots+\left(f_{n}\right)_{\infty}$ and such that $h$ satisfies the conditions in the statement of the lemma. 


\section{Algebraic points and algebraic distributions}

In this section we give the proofs of the theorems. We begin with Theorem 1.2 for which we shall give two different proofs. As usual the first step in the proofs is the construction of an auxiliary function $F$. Here we use Lemma 2.2. In the first proof we apply the maximum principle to the function $F / \phi_{N}$ with $\phi_{N}$ constructed in Lemma 3.3. The second proof is based on the Griffiths-King exhaustion function which was constructed in Lemma 3.4 and on Jensen's Formula associated with the exhaustion function.

Proof of Theorem 1.2. Let $f, g \in\left\{f_{1}, f_{2}, \ldots, f_{N}\right\}$ be algebraically independent over $K$. We define $m=|\mathcal{S}|$ and choose integers $r, n$ with $n$ sufficiently large such that

$$
r^{2} \geqslant 2 n m \log (n) \geqslant(r-1)^{2} .
$$

For the construction of the auxiliary function we consider the polynomial $P=\sum_{i, j=1}^{r} a_{i, j} S^{i} T^{j}$ with undetermined coefficients $a_{i, j}$. They will be chosen in $\mathcal{O}_{K}$ such that the system of $m n$ linear equations

$$
\sum_{i, j=1}^{r} a_{i, j} \nabla^{k}\left(f^{i} g^{j}\right)(w)=0
$$

for $0 \leqslant k<n$ and $w \in \mathcal{S}$ in $r^{2}$ unknowns $a_{i, j}$ is satisfied. By Lemma 2.3 we see that the $L^{2}$-heights of the linear forms

$$
L_{k, w}=\sum_{i, j=1}^{r} a_{i, j} \nabla^{k}\left(f^{i} g^{j}\right)(w)
$$

in the unknowns $a_{i, j}$ can be estimated from above by

$$
[K: \mathbb{Q}] k \log (r+k \omega)+C(k+r) .
$$

On applying Lemma 2.2 with $N=r^{2}$ and $M=n m$ together with (2) and on observing that $r=$ $O(\sqrt{n \log (n)})$ which gives

$$
\frac{M}{N-M} \leqslant \frac{1}{\log (n)}
$$

for $n \geqslant 4$ we find a nontrivial solution $a=\left(\ldots, a_{i, j}, \ldots\right) \in \mathcal{O}_{K}^{r^{2}}$ such that

$$
h_{\max }^{+}(P) \leqslant C_{3}[K: \mathbb{Q}] n
$$

where $C_{3}$ only depends on $w$. Since $f, g$ are algebraically independent over $K$, the function $F=$ $P(f, g)$ is not identically zero.

From (13) we deduce that the holomorphic function $F$ on $C$ satisfies $(F)_{0} \geqslant \sum_{w \in \mathcal{S}} n(w)$. We let $s \geqslant n$ be the largest integer such that

$$
(F)_{0} \geqslant \sum_{w \in \mathcal{S}} s(w)
$$

By definition $\nabla^{s} F$ does not vanish at some $w \in \mathcal{S}$. By Lemma 2.3 the height $h^{+}\left(\nabla^{s} F(w)\right)$ is at most $h_{\text {max }}^{+}(P)+[K: \mathbb{Q}] s \log (r+s)+O(r+s)$ and this leads to

$$
h^{+}\left(\nabla^{s} F(w)\right) \leqslant[K: \mathbb{Q}] s \log s+C_{4} s
$$

where $C_{4}$ only depends on $\Delta,[K: \mathbb{Q}]$ and $w$. 
Variant 1. In this variant we obtain a bound which is slightly weaker than the bound stated in the theorem. We write $m=l m_{1}+t$ with $m_{1} \geqslant 0$ and $2 g \leqslant t \leqslant l+2 g-1$ and we may assume that $m_{1} \geqslant 1$ since otherwise $|\mathcal{S}| \leqslant 2 g+l-1$. Lemma 3.3 gives a function $\phi_{s}$ such that

$$
E=\frac{F}{\phi_{s}}
$$

is holomorphic. We shall derive an upper bound by the maximum principle and by the Liouville estimate a lower bound for $\log |E(w)|$ and compare the upper and the lower bound. This will eventually give an estimate from above for $|\mathcal{S}|$ which is slightly weaker than (1).

We begin with the upper bound. Since $f$ and $g$ are of order $\leqslant \rho$ we know that for all $\epsilon>0$, for a sufficiently small positive $\eta$ and for all $p \in \mathcal{P}$ the inequality

$$
\log \max \left(\left|f\left(z_{p}\right)\right|,\left|g\left(z_{p}\right)\right|\right) \leqslant\left|z_{p}\right|^{-(\rho+\epsilon)}
$$

for $\left|z_{p}\right| \leqslant \eta$ gives

$$
\log \left|F\left(z_{p}\right)\right| \leqslant C_{5} n+2 r\left|z_{p}\right|^{-(\rho+\epsilon)} .
$$

Together with (ii) in Lemma 3.3 we conclude that

$$
\log \left|E\left(z_{p}\right)\right| \leqslant C_{5} n+2 r\left|z_{p}\right|^{-(\rho+\epsilon)}-m_{1} s \log \frac{C_{1}}{\left|z_{p}\right|} .
$$

From the maximum principle applied to the complement of the union of the discs with radius $\eta$ around $p$ for $p \in \mathcal{P}$ we get the upper bound

$$
\log |E(w)| \leqslant C_{5} n+2 r \eta^{-(\rho+\epsilon)}-m_{1} s \log \frac{C_{1}}{\eta} .
$$

For the lower bound we observe that $E(w)=\nabla^{s} F(w) / \nabla^{s} \phi_{s}(w)$ and that by (iii) in Lemma 3.3 we have

$$
\log \left|\nabla^{s} \phi_{s}(w)\right| \leqslant s \log s+s \log C_{2} .
$$

This leads together with Lemma 2.1 to the lower bound

$$
\log \left|\nabla^{s} F(w)\right| \geqslant-([K: \mathbb{Q}]-1) s \log s-C_{4} s .
$$

Note that the contribution involving $s \log s$ comes from the exponents of the polynomial $F$ by differentiating $s$ times a monomial, and therefore is in $\mathbb{Z}$. As a consequence one has only to account for $[K: \mathbb{Q}]-1$ terms of size $s \log s$.

We put the estimates together and obtain the lower bound

$$
\log |E(w)|=\log \left|\nabla^{s} F(w)\right|-\log \left|\nabla^{s} \phi_{s}(w)\right| \geqslant-[K: \mathbb{Q}] s \log s-C_{6} s .
$$

Since $n \leqslant s$ a comparison of the upper bound (16) and the lower bound above gives

$$
-[K: \mathbb{Q}] s \log s \leqslant 2 r \eta^{-(\rho+\epsilon)}-m_{1} s \log \frac{C_{1}}{\eta}+C_{7} s,
$$

where $C_{7}$ only depends on $w,[K: \mathbb{Q}]$ and $\Delta$. We relate $\eta$ and $s$ by the equation $s \eta^{\rho+\epsilon}=r C_{1}^{\rho+\epsilon}$ and find that

$$
0 \leqslant[K: \mathbb{Q}] s \log s-\left(\frac{m_{1}}{2 \rho+2 \epsilon}\right) s \log s+C_{8} s \log \log s .
$$

This can hold for large $s$ only if $[K: \mathbb{Q}](\rho+\epsilon) \geqslant m_{1}$ for all $\epsilon>0$ so that

$$
|\mathcal{S}|=m \leqslant l\left(m_{1}+1\right)+2 g-1 \leqslant 2[K: \mathbb{Q}] \rho l+l+2 g-1 \leqslant l+2[K: \mathbb{Q}] \rho l+2 g-1 .
$$


REMARK. Note that the upper bound can be improved to $2[K: \mathbb{Q}] \rho l+2 g-1$ if $\rho \geqslant(l-1) / 2 l$.

Variant 2. In this variant we use Jensen's Formula which needs an exhaustion function. We fix a positive integer $q$ and then Lemma 3.4 gives a projection $\pi: Z \rightarrow \mathbb{P}^{1}$ with $q l \leqslant \operatorname{deg} \pi \leqslant q l+g$. Furthermore the Lemma shows that there exists a divisor $D \geqslant 0$ such that $(\pi)_{\infty}=\sum_{p \in \mathcal{P}} q(p)+D$. We put $C^{\prime}:=C \backslash \operatorname{supp} D$ and replace $\mathcal{P}$ by $\mathcal{P}^{\prime}=\operatorname{supp} D \supseteq \mathcal{P}, \mathcal{S}$ by $\mathcal{S}^{\prime}=\mathcal{S} \backslash \operatorname{supp} D$ to obtain a new curve such that $\pi$ restricts to a finite covering $\pi^{\prime}: C^{\prime} \rightarrow \mathbb{C}$ which is totally ramified above 0 with $\pi^{\prime-1}(0)=t(w)$ for $t=\operatorname{deg} \pi$.

Similar to the definition of $E$ we define the function $G=F^{t} / \pi^{s}$ on $C^{\prime}$ with $s$ the order of $F$ at $w$. The function $G$ is holomorphic on $C^{\prime}$ by construction and has divisor

$$
D_{0} \geqslant s t \sum_{u}(u)
$$

with the sum taken over all $u \neq w$ in $\mathcal{S}^{\prime}$.

We choose a local parameter $z$ at $w$ such that $z(w)=0$ and such that $\nabla$ can be written as $\partial / \partial z$. Then we have $F(z)^{t} / z^{s t}=\left(\nabla^{s} F(w) / s !\right)^{t}+z \epsilon(z)$ and $\pi(z)^{s} / z^{s t}=\epsilon^{\prime}(z)^{s}$ near $w$ where $\epsilon$ and $\epsilon^{\prime}$ are units. This gives

$$
G(w)=\frac{F(w)^{t}}{\pi(w)^{s}}=\frac{\left(\nabla^{s} F(w) / s !\right)^{t}}{\epsilon^{\prime}(0)^{s}}
$$

and the lower bound

$$
\log |G(w)| \geqslant-([K: \mathbb{Q}]-1) t s \log s-C_{9} t s
$$

follows readily. The constant $C_{9}$ only depends on $w$ and $q$.

Now we are ready to apply Jensen's Formula (9) with $C$ replaced by $C^{\prime}$ and $f$ replaced by $G$. Since $\pi$ is totally ramified in $w$ the sum $\sum_{\pi(z)=0} \log |G(z)|$ in Jensen's Formula becomes $t \log |G(w)|$ and from the inequality above we obtain for the sum the lower bound

$$
\sum_{\pi(z)=0} \log |G(z)| \geqslant-([K: \mathbb{Q}]-1) t^{2} s \log s-C_{9} t^{2} s .
$$

For the integral we need an estimate from above for $|G|_{R}:=\max (|G(z)|)$ where the maximum is taken over all $z \in C^{\prime}$ with $|\pi(z)|=R$. The boundary $\Gamma$ decomposes into connected components $\Gamma_{p}$, one for each $p \in \operatorname{supp}(\pi)_{\infty}$ for which we choose local coordinates $z_{p}=(1 / \pi(z))^{1 / n_{p}}$. We have

$$
\max _{z \in \Gamma_{p}}(\log |G(z)|)=t \max _{z \in \Gamma_{p}}(\log |F(z)|)-s \log R .
$$

Since $n_{p} \geqslant q$ for $p \in \mathcal{P}$ the inequality (16) gives

$$
\max _{z \in \Gamma_{p}}(\log |G(z)|) \leqslant C_{5} t n+2 r t R^{(\rho+\epsilon) / q}-s \log R
$$

for $p \in \mathcal{P}$. This also holds for $p \in \operatorname{supp}(\pi)_{\infty} \backslash \mathcal{P}$ since $f$ and $g$ are of order 0 at $p$. We conclude that

$$
\int_{\partial C^{\prime}[R]} \log |G|^{2} d^{c} \tau \leqslant C_{5} t^{2} n+2 r t^{2} R^{(\rho+\epsilon) / q}-s t \log R .
$$

We also need a lower bound for the zero divisor $D_{0}$ of the holomorphic function $G$ on $C^{\prime}$. Since $\left|C \backslash C^{\prime}\right| \leqslant g$ by Lemma 3.4 the number of terms in the sum is at least $m-g-1$ and this leads to the lower bound

$$
n^{\pi}\left(D_{0}, r\right)=\int_{D[r]} 1 \geqslant s t(m-g-1)
$$


for sufficiently large $r$. Therefore we obtain

$$
N^{\pi}\left(D_{0}, R\right) \geqslant(m-g-1) s t \log \frac{R}{C_{10}}
$$

for some positive constant $C_{10}$ which depends only on $w$ and $q$ provided that $R$ is sufficiently large which is certainly the case if $r R^{(\rho+\epsilon) / q}=s$. This choice makes the estimate for the integral given in (19) a second order term of the form $O\left(t^{2} s\right)$. A comparison of the leading terms for $n$ and therefore also for $s$ going to infinity in (18)-(20) shows that

$$
(m-g-1) q \leqslant 2[K: \mathbb{Q}](\rho+\epsilon) t
$$

for all $q \geqslant 1$. Since by (i) in Lemma 3.4 we have $\lim _{q \rightarrow \infty} t / q=l$ the desired inequality for $|\mathcal{S}|$ follows readily.

REMARK. By a suitable modification of Lemma 3.4 and of the second proof of Theorem 1.2 the statement of Theorem 1.2 can be improved to give

$$
|\mathcal{S}| \leqslant g+1+2[K: \mathbb{Q}] \sum_{p \in \mathcal{P}} \rho_{p},
$$

where $\rho_{p}=\max _{i} \rho_{p}\left(f_{i}\right)$.

Proof of Theorem 1.3. Let $\mathcal{S} \subset C$ be a finite set such that $f(\mathcal{S}) \subset \mathcal{U}(K)$. Let $\pi: \widetilde{X} \rightarrow X$ be the blow-up of $X$ in $X \backslash \mathcal{U}$ and let $E=\pi^{-1}(X \backslash \mathcal{U})$ be the exceptional divisor. We choose a hyperplane section $H$ of $X$ which does not meet $f(\mathcal{S})$ and put $\widetilde{H}=\pi^{-1} H$. Then if $n$ is sufficiently large the divisor $n \widetilde{H}+E$ is very ample. Therefore $\widetilde{Y}=\widetilde{X} \backslash(\widetilde{H} \cup E)$ takes the form Spec $R$ for some $K$-algebra $R$ of finite type which can be written as $R=K\left[y_{1}, y_{2}, \ldots, y_{n}\right]$. Since the rational vector field $\Delta$ is regular on $\widetilde{Y}$ we have $\Delta\left(\mathcal{O}_{\widetilde{Y}, y}\right) \subseteq \mathcal{O}_{\widetilde{Y}, y}$ for all $y \in \widetilde{Y}$ and therefore $\mathcal{O}_{\widetilde{Y}, y}=R_{\mathfrak{m}_{y}}$ where $\mathfrak{m}_{y}$ denotes the maximal ideal at $y$. By [20; Theorem 4.7] we have $R=\bigcap_{y \in \widetilde{Y}} R_{\mathfrak{m}_{y}}$. We conclude that $\Delta(R) \subseteq R$ and hence there exist $g_{i} \in K\left[y_{1}, \ldots, y_{n}\right]$ such that $\Delta\left(y_{i}\right)=g_{i}$ on $\tilde{Y}$. Then the functions $f_{i}(z)=f^{*}\left(y_{i}\right)$ satisfy $\nabla\left(f_{i}\right)=g_{i}\left[f_{1}, \ldots, f_{n}\right]$. They are meromorphic of order $\leqslant \rho$ on $C$ and satisfy $f_{i}(w) \neq \infty$ for $1 \leqslant i \leqslant n$ and $w \in \mathcal{S}$. Let $h$ be as in Lemma 5.4 and replace in the second proof the function $G=F^{t} / \pi^{s}$ by $G=F^{t} h^{2 r t} / \pi^{s}$. The required estimate follows then similarly.

REMARK. Our method still works if the holomorphic tangent vector $\nabla$ has zeroes and we get results similar to Theorem 1.2 and Theorem 1.3. For instance the conclusion of Theorem 1.2 holds for $\mathcal{S}$ replaced by $\mathcal{S}_{0}=\left\{w \in \mathcal{S}: \nabla_{w} \neq 0\right\}$.

Proof of the Theorem 1.1. Our discussion before the statement of Theorem 1.1 shows that the hypothesis of Theorem 1.3 are satisfied. By Theorem 1.3,

$$
\left|f^{-1}(\mathcal{U}(K))\right| \leqslant g+1+2[K: \mathbb{Q}] l \max (\rho, 2 g) .
$$

However $C$ is integral and this implies that $\psi(C) \subseteq \mathcal{U}$ which implies desired results.

\section{Singularities}

It is critical to exclude poles in the formulation of Theorem 1.3. Otherwise the relation $\left|f^{-1}(X(K))\right|<\infty$ is not generally true. We give a simple example.

ExAmple 7.1. Consider the map $f: \mathbb{C} \rightarrow \mathbb{P}^{3}=\left\{\left(z_{0}: z_{1}: z_{2}: z_{3}\right)\right\}$ which is given by $f(z)=$ $\left(z: \wp(z): \wp^{\prime}(z): 1\right)$ and $\nabla \in \Gamma\left(\mathbb{C}, \mathcal{T}_{\mathbb{C}}\right)$ where $\nabla=\partial / \partial z$. Let

$$
z_{02}=\frac{z_{0}}{z_{2}}, \quad z_{12}=\frac{z_{1}}{z_{2}}, \quad z_{32}=\frac{z_{3}}{z_{2}} .
$$


Then we may take $\Delta$ as

$$
\begin{gathered}
\Delta\left(z_{02}\right)=z_{32}-\frac{z_{02} z_{12}^{2}}{z_{32}}+\frac{g_{2}}{2} z_{02} z_{12} \\
\Delta\left(z_{12}\right)=1-\frac{6 z_{12}^{3}}{z_{32}}+\frac{g_{2}}{2} z_{12} z_{32}, \quad \Delta\left(z_{32}\right)=\frac{g_{2}}{2} z_{32}^{2}-6 z_{12}^{2} .
\end{gathered}
$$

Notice that $A=(0: 0: 1: 0)$ is in the set $P(\Delta)$ of singular points of $\Delta$ and also in $\mathbb{P}^{3}(K)$ but $\left|f^{-1}(A)\right|=|\Lambda|=\infty$. This occurs because $A \notin \mathcal{U}(K)$.

A slight modification of the example leads to

ExAmple 7.2. Consider the map $f: \mathbb{C} \rightarrow X=V\left(z_{2}^{2} z_{3}-4 z_{1}^{3}+g_{2} z_{1} z_{3}^{2}+g_{3} z_{3}^{3}\right) \subset \mathbb{P}^{3}$ which is given by $f(z)=\left(z: \wp(z): \wp^{\prime}(z): 1\right)$ and $\nabla=\partial / \partial z \in \Gamma\left(\mathbb{C}, \mathcal{T}_{\mathbb{C}}\right)$. Now we have

$$
\begin{gathered}
\Delta\left(z_{02}\right)=z_{32}-\frac{z_{02} z_{12}^{2}}{z_{32}}+\frac{g_{2}}{2} z_{02} z_{12}, \\
\Delta\left(z_{12}\right)=1-\frac{6 z_{12}^{3}}{z_{32}}+\frac{g_{2}}{2} z_{12} z_{32}, \quad \Delta\left(z_{32}\right)=\frac{g_{2}}{2} z_{32}^{2}-6 z_{12}^{2} .
\end{gathered}
$$

Although $6 z_{12}^{3} / z_{32}$ is a regular function at $A=(0: 0: 1: 0) \in X, z_{02} z_{12}^{2} / z_{32}$ is not a regular function at $A \in X$. This explains why we have both $A=(0: 0: 1: 0) \in X(K)$ and $\left|f^{-1}(A)\right|=$ $|\Lambda|=\infty$.

We now change in a different way and give a slightly new proof of a theorem of Schneider on the transcendence of periods.

ExAmple 7.3. Consider the map $f: \mathbb{C} \rightarrow X=\mathbb{P}^{1} \times E$ which is given by $f(z)=((z: 1),(\wp(z):$ $\left.\left.\wp^{\prime}(z): 1\right)\right)$ where

$$
E=V\left(z_{2}^{2} z_{3}-4 z_{1}^{3}+g_{2} z_{1} z_{3}^{2}+g_{3} z_{3}^{3}\right) \subset \mathbb{P}^{2}=\left\{\left(z_{1}: z_{2}: z_{3}\right)\right\}
$$

and $\nabla=\partial / \partial z \in \Gamma\left(\mathbb{C}, \mathcal{T}_{\mathbb{C}}\right)$. One can check that $f_{*} \nabla$ is regular at $((\mathrm{z}: 1),(0: 1: 0))$ for all $z \in \mathbb{C}$. If a non-zero $w \in \Lambda$ is an algebraic number and is in $K$, then we will have $f(n w)=((n w: 1)$, $\left.(0: 1: 0)) \in X(K) \backslash P\left(f_{*} \Delta\right)\right)$ for all $n \in \mathbb{N}$. This contradicts Theorem 1.3.

\section{Appendix}

8.1. Schneider's Theorem revisited. In this appendix we shall state Schneider's theorem as announced in the introduction, we shall comment on it and relate it to our work. We make the same geometric assumptions as in Theorem 1.2. In particular we fix a vector field $\nabla$.

In Section 4 we introduced the notion of an analytic divisor on $C$ to be an element of the group Div $C=\lim _{\longrightarrow} \operatorname{Div} C[r]$. We shall extend the notion slightly and define $\widetilde{\operatorname{Div}} C$ using the same direct system and requiring that an element of $\widetilde{\operatorname{Div}} C$ is a family of divisors $D=\{D[r]=$ $\left.\sum_{z \in C[r]} D[r](z)(z)\right\}$ which as divisors satisfy $D[r] \leqslant D[s]$ for $r \leqslant s$. The limit is given by $\coprod \operatorname{Div} C[r] / \sim$ where $D[r] \sim D[s]$ if and only if there exists some $t$ such that $D[t] \geqslant D[r]$ and $D[t] \geqslant D[s]$. This obviously is an equivalence relation and shows that the limit exists.

Translated into the language of schemes this means that if $\mathcal{J}_{r}$ denotes the ideal of $D[r]$ in $\mathcal{O}_{C}$ then there are closed immersions $\iota[r]: \operatorname{Spec}\left(\mathcal{O}_{C} / \mathcal{J}_{r}\right) \rightarrow C$ which form a direct system of schemes and we write $\widetilde{D}=\lim _{\longrightarrow} \operatorname{Spec}\left(\mathcal{O}_{C} / \mathcal{J}_{r}\right)$. It has the property that the divisor associated with $\widetilde{D}$ is $D$. We identify $D[r]$ with $\operatorname{Spec}\left(\mathcal{O}_{C} / \mathcal{J}_{r}\right)$. Clearly we have $\widetilde{\operatorname{Div}} C \supseteq \operatorname{Div} C$. A divisor $D$ in $\widetilde{\operatorname{Div}} C$ is effective if $D[r] \geqslant 0$ for all $r$. There is for each $r$ a canonical surjective homomorphism of sheaves $\iota[r]^{\sharp}: \mathcal{O}_{C}^{\text {an }} \rightarrow \iota[r]_{*} \mathcal{O}_{D[r]}$. Here $\mathcal{O}_{C}^{\text {an }}$ is the sheaf of germs of holomorphic functions on $C$. The kernel of $\iota[r]^{\sharp}$ is an ideal sheaf $\mathcal{J}[r]$. For $z \in \operatorname{supp} D[r]$ we choose a local coordinate $\tau_{z} \in \mathcal{J}[r]_{z}$ at $z$ such 
that $\nabla=d / d \tau_{z}$ locally at $z$. The local coordinate is uniquely determined by this property. For $D[r]$ given we introduce the algebra $R[r]=\bigoplus K\left[\tau_{z}\right] /\left(\tau_{z}^{D[r](z)}\right)$ and then $\Gamma\left(D[r], \iota[r]_{*} \mathcal{O}_{D[r]}\right)=R[r] \otimes_{K} \mathbb{C}$. There is a canonical identification of $R[r]$ considered as a vector space with $K^{D[r]}$ and this is used to define the $h^{+}(f)$ of $f \in R[r]$. The homomorphism $\iota[r]^{\sharp}$ extends to the sheaf of germs of meromorphic functions on $C$ which are holomorphic on $D$. We let now $D \in \widetilde{\mathrm{Div}} C$ be a divisor and define

$$
\delta=\underline{\lim } \frac{\log \operatorname{deg} D[r]}{\log r} .
$$

Let $f$ be a meromorphic function on $C$ which is holomorphic on $D$ and which has the property that $\iota[r]^{\sharp}(f) \in R[r]$ for all $r$. Then the arithmetic growth of $f$ along $D$ is defined as

$$
\mu=\varlimsup \frac{\log h^{+}\left(\iota[r]^{\sharp}(f)\right)}{\log \operatorname{deg} D[r]} .
$$

Let $D \in \widetilde{\mathrm{Div}} C$ be an effective divisor such that $D[r](z) \log D[r](z) \leqslant \operatorname{deg} D[r]$ for all $z \in$ $\operatorname{supp} D[r]$. Let $f_{1}, f_{2}, \ldots, f_{n}$ be holomorphic functions on $C$ and assume that $\iota[r]^{\sharp}\left(f_{i}\right) \in R[r]$ for $1 \leqslant i \leqslant n$ and $r$ sufficiently large. We define $\rho=\max _{i}\left(\rho_{i}\right)$ and $\mu=\max _{i}\left(\mu_{i}\right)$ where $\rho_{1}, \rho_{2}, \ldots, \rho_{n}$ and $\mu_{1}, \mu_{2}, \ldots, \mu_{n}$ are the growth and arithmetic growth respectively of the functions. The following theorem is an extension to Riemann surfaces of Schneider's Satz III in [5] mentioned in the introduction in the case when the functions are holomorphic.

THEOREM 8.1. If we have $\max (\mu, \rho / \delta)<1-1 / n$ then the image of the mapping

$$
f=\left(f_{1}, f_{2}, \ldots, f_{n}\right): C \rightarrow \mathbb{C}^{n}
$$

is contained in an algebraic hypersurface defined over $K$.

The proof is very similar to the proof of our main theorems but here (8) is not sufficient, instead we need to use a version of Jensen's Formula as formulated in [1; p. 332]. In the case when the functions are meromorphic one has to assume in addition that $\rho \geqslant 2 g$ where $g$ is the genus of $C$. This is needed because [17; Proposition 5.25] (see Section 8.3).

As an application which is not covered by any of the theorems in the introduction we take a meromorphic function $f$ on $C$ which has the property that $\iota[r]^{\sharp}(f) \in R[r]$ for all sufficiently large $r$. We put $f_{1}=f, f_{2}=\nabla f, \ldots, f_{n}=\nabla^{n-1} f$ for $n$ sufficiently large so to satisfy the hypothesis of the theorem. Then the function $f$ satisfies an algebraic differential equation with constant coefficients. As has been mentioned in the introduction the theorem does not include Gelfond's proof of the seventh Hilbert Problem and, as a consequence, does not include the Schneider-Lang Criterion, even in the case when $C=\mathbb{C}$. It would be very interesting to find a criterion in the style of our theorem above which does include our main theorems and without making the assumption that the functions satisfy differential equations.

All theorems that have been mentioned so far deal only with the transcendence of numbers. As already has been pointed out in the last paragraph of Schneider's paper in [5] the methods are not strong enough to get a criterion about algebraic independence. The only substantial contribution in this very general direction is [21]. The techniques which are applied there are much more involved. Any further progress in the direction opened there would be of highest interest.

8.2. Proof of Theorem 8.1. We shall give now a short sketch of the proof of the theorem. The proof is very similar to the proof of Theorem 1.2 and depends on estimates of the same flavor as those given there together with estimates similar to those in [5]. Therefore we shall not give such details here which can be extracted without much effort from [5]. The main difference is that we do not use any kind of Schwarz Lemma. Especially in the case when the functions are meromorphic but not holomorphic the estimates which are needed there become very unpleasant. Instead our approach goes through Nevanlinna theory and seems to be new. We reduce the proof 
to an application of Jennsen's Formula together with the First Main Theorem. This gives a very elegant and clean way to do the last step in the proof. For simplicity we restrict ourselves to the special case when $C=\mathbb{C}$.

The first step is standard. We choose an integer $r$ sufficiently large and construct on the basis of Siegel's Lemma a polynomial $F \in K\left[T_{1}, \ldots, T_{n}\right]$ of degree $d$ such that $f \in \operatorname{ker} \iota[r]^{\sharp}$ for $f=$ $F\left(f_{1}, \ldots, f_{n}\right)$ and $\iota$ taken in the analytic category. The parameters are chosen such that

$$
(d+1)^{n} \geqslant N(D[r], R)>d^{n}
$$

and such that $R=r^{1+\epsilon}$. As a consequence we have

$$
N(D[r], R) \geqslant N(D[r], r)+m(D[r]) \log \left(\frac{R}{r}\right)
$$

where $m=m(D[r])$ denotes the geometric degree of the divisor $D[r]$. This shows that the exponent $M /(N-M)$ which occurs in Siegel's Lemma is of order $1 / \log m$. An application of the Siegel lemma shows that the logarithmic height of $F$ is at most $O\left(m^{1 / n+\mu+\epsilon}+m^{1 / n+\eta+\epsilon}\right)$ since the exponent $M /(N-M)$ kills the $\log m$ in the logarithm of the factorials. The condition on $\eta$ then shows that this is at most $O\left(m^{1-\epsilon}\right)$ for $\epsilon$ sufficiently small. The function $f$ is in ker $\iota[r]^{\sharp}$ by construction and this shows that $N(f, 0, R) \geqslant m(D[r]) \log (R / r)$. We write $f(z)=c_{e} z^{e} \epsilon(z)$ with $\epsilon(z)=1+\sum_{n \geqslant 1} \epsilon(n) z^{n}$ and assume for simplicity that 0 is a component of the divisor. The general case can be treated in a similar way.

Lemma 8.2 (Jensen's Inequality). Let $f$ be meromorphic. Then

$$
\log \left|c_{e}\right| \leqslant T(f, \infty, r)-N(f, 0, r) .
$$

Proof. This is an easy consequence of Jensen's Formula.

In our situation $N(f, 0, R)$ can be bounded from below by $N(D[r], r)$, we use standard inequalities for the Nevanlinna characteristic function $T$ to bound $T(f, \infty, R)$ from above and the Liouville estimate gives a lower bound for $\log \left|c_{e}\right|$. Comparing the upper and lower bound then

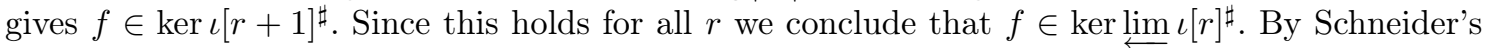
arguments one deduces that $f=0$.

8.3. Meromorphic functions of finite growth on affine varieties. In Theorems 1.1 and 1.3 the upper bound for the number of points in the set $S$ contains the term $\max (\rho, 2 g)$ with $g$ the genus of the affine curve $C$. It is an interesting question to see whether the dependence is natural or whether it can be removed. This turns out to be a question about meromorphic functions on affine curves.

The field of meromorphic functions $\mathcal{M}(C)$ contains as a subfield the field of meromorphic functions $\mathcal{M}_{\text {f.o. }}(C)$ of finite order with the order defined by using either the Nevanlinna or the Ahlfors-Schimizu characteristic function. Both characteristic functions are based on a choice of an exhaustion function. In Section 4 such a function has been constructed on applying the Riemann-Roch Theorem. We have seen that the resulting order does not depend on the chosen characteristic function. The field $\mathcal{M}_{\text {f.o. }}(C)$ contains for any $\rho$ as algebraically closed subfield the field $\mathcal{M}_{\rho}(C)$ of meromorphic functions of order at most $\rho$ and is the direct limit of this system of subfields (see [22]).

A basic question is whether there are meromorphic functions on an affine curve $C$ different from algebraic functions. Another question is whether a meromorphic function of order $\rho$ can be expressed as a quotient of two entire functions of order $\rho$. It turns out that this is the case provided that the order is $\geqslant 2 g$ (see Proposition 5.2) and the bound is also best possible in general as can be found in the already mentioned articles of Griffiths.

The answer to the first question is given by the Weierstrass Product Theorem on Riemann surfaces. In section 4 we introduced the analytic divisors $\operatorname{Div}(C)$ on $C$ which can be seen as 
maps $D: C \rightarrow \mathbb{Z}$. By [23; Theorem 25.5] each divisor $D$ is principal and this shows that there are meromorphic functions $f$ on $C$ which have $D$ a divisor. If the divisor $D$ is effective so that $D(z) \geqslant 0$ for all $z \in C$ then we introduced $N^{\pi}(D, r)$ and $D$ has order $\rho$ if $N^{\pi}(D, r)=O\left(r^{\rho}\right)$. By [17; Theorem I] there exists an entire function $f$ on $C$ which has $\rho(f)=\rho(D)$ and divisor $D$. As an application a transcendental function of finite order can be constructed by choosing a divisor $D$ with $N^{\pi}(D, r)=O\left(r^{\rho}\right)$ and $\rho>0$ and then the function $f$ is transcendental. An interesting consequence is the following.

TEOPEMA 8.1. For each non-negative integer $n$ there exists a subring $L \subseteq \mathcal{M}_{\text {f.o. }}(C)$ which has transcendence degree $n$ over the field of rational functions $\mathcal{R}(C)$ of $C$.

Proof. For $n=0$ we take $L_{0}=\mathcal{R}(C)$. We proceed by induction and assume the theorem to be true for $n$ with subring $L_{n}$. There exist $f_{1}, \ldots, f_{n} \in L_{n}$ such that $L_{n}$ is algebraic over $\mathcal{R}(C)\left[f_{1}, \ldots, f_{n}\right]$. We take $\rho=\max _{1 \leqslant i \leqslant n}\left(\rho\left(f_{i}\right)\right)$ and choose $f_{n+1} \in \mathcal{O}_{k}(C)$ with $k=\rho\left(f_{n+1}\right)>\rho$. Since $\mathcal{O}_{\rho}(C)$ is algebraically closed in $\mathcal{M}_{\rho}(C)$ the entire function $f_{n+1}$ is transcendental over $L_{n}$ and we tale $L_{n+1}=L_{n}\left[f_{n+1}\right]$.

The authors would like to thank P. Ng from HKU for carefully going through the first version of this paper which was part of the thesis of the first author at ETHZ in 2011 and for carefully reading the revised and expanded final version. The second author likes to thank the Department of Mathematics of the Hong Kong University of Science and Technology for inviting him in Summer 2010 to give a series of lectures on the first version of the paper. The final version was finished in summer 2012 during a stay as a visiting professor at the Hong Kong University.

\section{Bibliography}

[1] S.-s. Chern, "Complex analytic mappings of Riemann surfaces I", Amer. J. Math., 82:2 (1960), 323-337.

[2] A. Gelfond, "Sur le septième problème de Hilbert", Известия Академии наук СССР. VII серия. Отделение математических и естественных наук, 1934, № 4, 623-634.

[3] Th. Schneider, "Transzendenzuntersuchungen periodischer Funktionen. I. Transzendenz von Potenzen", J. Reine Angew. Math., 172 (1934), 65-69.

[4] Th. Schneider, "Transzendenzuntersuchungen periodischer Funktionen. II. Transzendenzeigenschaften elliptischer Funktionen", J. Reine Angew. Math., 172 (1934), 70-74.

[5] Th. Schneider, "Ein Satz über ganzwertige Funktionen als Prinzip für Transzendenzbeweise", Math. Ann., 121:1 (1949), 131-140.

[6] S. Lang, Introduction to Transcendental Numbers, Addison-Wesley Publ., Reading, MA, 1966.

[7] E. Bombieri, "Algebraic values of meromorphic maps", Invent. Math., 10:4 (1970), 267-287.

[8] R. Nevanlinna, Analytic Functions, Grundlehren Math. Wiss., 162, Springer-Verlag, Berlin, 1970.

[9] W. Stoll, "Die beiden Hauptsätze der Wertverteilungstheorie bei Funktionen mehrerer komplexer Veränderlichen (I)", Acta Math., 90 (1953), 1-115; "Die beiden Hauptsätze der Wertverteilungstheorie bei Funktionen mehrerer komplexer Veränderlichen (II)", Acta Math., 92 (1954), 55-169.

[10] S.-s. Chern, "The integrated form of the first main theorem for complex analytic mappings in several complex variables", Ann. of Math. (2), 71:3 (1960), 536-551.

[11] J. Carlson, P. Griffiths, "A defect relation for equidimensional holomorphic mappings between algebraic varieties", Ann. of Math. (2), 95:3 (1972), 557-584.

[12] P. Griffiths, J. King, "Nevanlinna theory and holomorphic mappings between algebraic varieties", Acta Math., 130 (1973), 145-220.

[13] I. Wakabayashi, "Algebraic values of meromorphic functions on Riemann surfaces", J. Number Theory, $\mathbf{2 5 : 2}$ (1987), 220-229.

[14] L. Hörmander, " $L^{2}$ estimates and existence theorems for the $\bar{\partial}$ operator", Acta Math., 113 (1965), 89-152. 
[15] E. Bombieri, J. Vaaler, "On Siegel's lemma", Invent. Math., 73:1 (1983), 11-32.

[16] A. Baker, G. Wüstholz, Logarithmic Forms and Diophantine Geometry, New Math. Monogr., 9, Cambridge Univ. Press, Cambridge, 2007.

[17] P. A. Griffiths, "Function theory of finite order on algebraic varieties. I(A)", J. Differential Geometry, 6 (1972), 285-306; "Function theory of finite order on algebraic varieties. I(B)", J. Differential Geometry, 7 (1972), 45-66.

[18] P. Griffiths, Entire Holomorphic Mappings in One and Several Complex Variables, Ann. of Math. Stud., 85, Princeton Univ. Press, Princeton, NJ, 1976.

[19] R. Narasimhan, Several Complex Variables, Chicago Lectures in Math., The Univ. of Chicago Press, Chicago, IL, 1971.

[20] H. Matsumura, Commutative Ring Theory, Cambridge Stud. Adv. Math., 8, Cambridge Univ. Press, Cambridge, 1989.

[21] G. Wüstholz, "Algebraische Unabhängigkeit von Werten von Funktionen, die gewissen Differentialgleichungen genügen", J. Reine Angew. Math., 317 (1980), 102-119.

[22] S. Hellerstein, L. A. Rubel, "Subfields that are algebraically closed in the field of all meromorphic functions", J. Analyse Math., 12 (1964), 105-111.

[23] O. Forster, Lectures on Riemann Surfaces, Grad. Texts in Math., 81, Springer-Verlag, Berlin, 1981. 